\title{
Comparative Analysis of Context-Dependent Mutagenesis in Humans and Fruit Flies
}

\author{
Sofya A. Medvedeva, ${ }^{1}$ Alexander Y. Panchin, ${ }^{2}$ Andrey V. Alexeevski, ${ }^{1,3,4}$ \\ Sergey A. Spirin, ${ }^{1,3,4}$ and Yuri V. Panchin ${ }^{2,3}$ \\ ${ }^{1}$ Department of Bioengineering and Bioinformatics, Moscow State University, Vorbyevy Gory 1-73, Moscow 119992, Russia \\ ${ }^{2}$ Institute for Information Transmission Problems, Russian Academy of Sciences, Bolshoi Karetny pereulok 19-1, \\ Moscow 127994, Russia \\ ${ }^{3}$ Department of Mathematical Methods in Biology, Belozersky Institute, Moscow State University, Vorbyevy Gory 1-40, \\ Moscow 119991, Russia \\ ${ }^{4}$ Department of Mathematics, Scientific-Research Institute for System Studies, Russian Academy of Sciences, \\ Nakhimovskii prospekt 36-1, Moscow 117218, Russia
}

Correspondence should be addressed to Alexander Y. Panchin; alexpanchin@yahoo.com

Received 1 April 2013; Accepted 7 July 2013

Academic Editor: Dmitry Sherbakov

Copyright (C) 2013 Sofya A. Medvedeva et al. This is an open access article distributed under the Creative Commons Attribution License, which permits unrestricted use, distribution, and reproduction in any medium, provided the original work is properly cited.

\begin{abstract}
In general, mutation frequencies are context-dependent: specific adjacent nucleotides may influence the probability to observe a specific type of mutation in a genome. Recently, several hypermutable motifs were identified in the human genome. Namely, there is an increased frequency of T>C mutations in the second position of the words ATTG and ATAG and an increased frequency of $\mathrm{A}>\mathrm{C}$ mutations in the first position of the word ACAA. Previous studies have also shown that there is a remarkable difference between the mutagenesis of humans and drosophila. While $\mathrm{C}>\mathrm{T}$ mutations are overrepresented in the CG context in humans (and other vertebrates), this mutation regularity is not observed in Drosophila melanogaster. Such differences in the observed regularities of mutagenesis between representatives of different taxa might reflect differences in the mechanisms involved in mutagenesis. We performed a systematical comparison of mutation regularities within 2-4 bp contexts in Homo sapiens and Drosophila melanogaster and found that the aforementioned contexts are not hypermutable in fruit flies. It seems that most mutation contexts affect mutation rates in a similar manner in H. sapiens and D. melanogaster; however, several important exceptions are noted and discussed.
\end{abstract}

\section{Introduction}

The average rates of point mutations in multicellular eukaryotic genomes are usually between $10^{-7}$ and $10^{-10}$ mutations per nucleotide per generation $[1,2]$. However, the rates of point mutations may be dramatically altered by their genomic context. In some cases, this context-dependent change in mutation frequency can be attributed to known molecular mechanisms involved in mutagenesis. For example, the increased frequency of $\mathrm{C}>\mathrm{T}$ mutations in the word $\mathrm{CG}$ in humans (and other vertebrates) is attributed to the methylation of cytosines by context-specific DNA methyltransferases [3]. This mutation regularity is absent in D. melanogaster [4], in which cytosine methylation occurs, but appears to be restricted to early embryonic development and is not specific to cytosines followed by guanines [5]. Many other examples of context-dependent mutagenesis have been reported $[4,6-$ 9].

Recently, an increased rate of $\mathrm{T}>\mathrm{C}$ mutations in the second position of the words ATTG and ATAG and an increased rate of $\mathrm{A}>\mathrm{C}$ mutations in the first position of the ACAA word were reported in the human genome [10]. This was achieved by calculating the values called "minimal contrast" and "mutation bias" for 2-4 bp mutation contexts to evaluate if the addition of specific nucleotides to the $5^{\prime}$ or $3^{\prime}$ end of $1-3$ bp words increases the probability of observing certain mutations in fixed positions. Mutation bias indicates the total excess (or deficiency) of mutations within a given 
context. Minimal contrast indicates the excess (or deficiency) of mutations within a given context that cannot be explained by the excess (or deficiency) of mutations in one of its subcontexts.

$H$. sapiens and D. melanogaster are perspective model organisms for this kind of studies because of the vast amount of data on genetic variation that is available for them. The goal of our study was to compare the mutation regularities of $H$. sapiens and D. melanogaster in terms of "minimal contrast" and "mutation bias."

\section{Methods}

We searched for single nucleotide variable positions in intergenic sequences of 37 individual $D$. melanogaster genomes (multiple alignments obtained from http://genome.ucsc.edu/ [11]). Drosophila sechellia (droSecl, Oct. 2005) and Drosophila erecta (droEre2, Feb. 2006) genomic sequences were used as outgroups to reconstruct the ancestral states for the variable positions. D. melanogaster genome (dm3, Apr. 2006) was used as the reference.

2.1. Mutation Data. We assume that a mutation with a known direction within a known context has occurred in a specific position of the $D$. melanogaster genome if the following conditions are met.

(1) D. sechellia and D. erecta genomes have the same nucleotide aligned to this position (this nucleotide will be referred to as the "ancestral nucleotide").

(2) Among the 37 D. melanogaster genomes, some contain the ancestral nucleotide in this position, while some other genomes contain a different nucleotide.

(3) Only 2 genetic variants are present in this position for the 37 D. melanogaster genomes.

(4) The $3 \mathrm{bp}$ upstream and downstream positions from these positions in the multiple alignment do not contain any substitutions or gaps.

Mutation bias and minimal contrasts for D. melanogaster were calculated for 2-4 bp mutation contexts using the methods described in [10]. Mutation bias, contrasts, and other data for H. sapiens were taken directly from [10].

2.2. Mutation Context and Subcontext. We denote the mutation context of mutation mut in position pos of the word W as $\{$ mut $\mid$ pos, $\mathrm{W}\}$. For example, $\{\mathrm{C}>\mathrm{T} \mid 1, \mathrm{CG}\}$ represents a $\mathrm{C}>\mathrm{T}$ mutation in the first position of the word CG. Mutation context $\left\{\right.$ mut $\mid$ pos $\left.^{\prime}, \mathrm{W}^{\prime}\right\}$ is called a subcontext of the context $\{$ mut $\mid$ pos, $\mathrm{W}\}$ if $\mathrm{W}^{\prime}$ is a subword of $\mathrm{W}$, and any mutation mut occurring in position pos of the word $\mathrm{W}$ is at the same time a mutation occurring in position pos' of the word $\mathrm{W}^{\prime}$. For example, $\{\mathrm{C}>\mathrm{T} \mid 1, \mathrm{CG}\}$ is a subcontext of $\{\mathrm{C}>\mathrm{T} \mid 2, \mathrm{ACG}\}$.
2.3. Contrast. For each pair of context $\{$ mut | pos, W $\}$ and its subcontext $\left\{\right.$ mut $\mid$ pos $\left.{ }^{\prime}, \mathrm{W}^{\prime}\right\}$, the value of contrast is given by the formula

$$
\begin{aligned}
& \text { Contrast }\left(\{\text { mut } \mid \text { pos, } \mathrm{W}\},\left\{\text { mut } \mid \text { pos }^{\prime}, \mathrm{W}^{\prime}\right\}\right) \\
& =\frac{P_{\{\text {mut } \mid \text { pos }, \mathrm{W}\}}}{P_{\left\{\text {mut } \mid \text { pos }{ }^{\prime}, \mathrm{W}^{\prime}\right\}}} \text {. }
\end{aligned}
$$

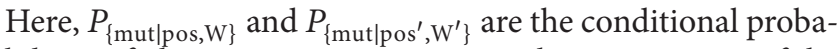
bilities of observing mutation mut in the position pos of the word $\mathrm{W}$ and position pos' of word $\mathrm{W}^{\prime}$, respectively, in a given dataset. Although these probabilities cannot be explicitly calculated without assumptions of the general probability of mutation per nucleotide in the genome, their ratio can be estimated by the following formula:

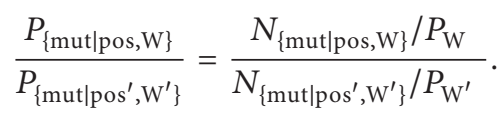

Here, $P_{\mathrm{W}}$ and $P_{\mathrm{W}^{\prime}}$ are the observed frequencies of words $\mathrm{W}$ and $\mathrm{W}^{\prime}$, respectively, among all words of the same length.

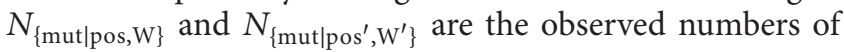
mutation mut in position pos of word $\mathrm{W}$ and position pos' of the word $\mathrm{W}^{\prime}$, respectively.

The ratio $P_{\mathrm{W}} / P_{\mathrm{W}^{\prime}}$ estimates the probability for $\mathrm{W}^{\prime}$ to be extended to $\mathrm{W}$. This ratio coincides with the expected ratio $N_{\{\text {mut|pos,W\} }} / N_{\left\{\text {mut|pos', } W^{\prime}\right\}}$ under the hypothesis that mutations rates are the same in the context $\{$ mut | pos, W\} and its subcontext $\left\{\right.$ mut $\mid$ pos $\left.^{\prime}, \mathrm{W}^{\prime}\right\}$. Therefore, if Contrast (\{mut | pos, $\mathrm{W}\},\left\{\right.$ mut $\left.\mid \operatorname{pos}^{\prime}, \mathrm{W}^{\prime}\right\}$ ) is greater than 1 , it indicates an increased mutation rate in the context \{mut | pos, $W\}$ compared with the subcontext $\left\{\right.$ mut | pos', $\left.\mathrm{W}^{\prime}\right\}$; while if Contrast ( $\{$ mut $\mid$ pos, $W\},\left\{\right.$ mut $\left.\left.\mid \operatorname{pos}^{\prime}, \mathrm{W}^{\prime}\right\}\right)$ is less than 1 , it indicates a decreased mutation rate.

2.4. Minimal Contrast. For a given context $\{$ mut | pos, W\}, let us consider all of its subcontexts $\left\{\right.$ mut $\mid$ pos' $\left.{ }^{\prime} \mathrm{W}^{\prime}\right\}$. The minimal contrast is the value $\mathrm{MC}=$ Contrast $(\{$ mut $\mid$ pos, $\mathrm{W}\},\left\{\right.$ mut $\left.\left.\mid \operatorname{pos}^{\prime}, \mathrm{W}^{\prime}\right\}\right)$ such that the absolute difference $|\mathrm{MC}-1|$ is the lowest among all subcontexts $\left\{\right.$ mut $\mid$ pos $\left.{ }^{\prime}, \mathrm{W}^{\prime}\right\}$. We did not study discontigvous contexts such as CNG and CNNG.

2.5. Mutation Bias. For any context $\{$ mut | pos, $\mathrm{W}\}$, there exists only one subcontext $\left\{\right.$ mut | $\left.\operatorname{pos}^{\prime}, \mathrm{W}^{\prime}\right\}$ such that the length of $\mathrm{W}^{\prime}$ is equal to 1 (i.e., $\mathrm{W}^{\prime}$ is the one-letter word consisting of the mutated letter). The mutation bias is the contrast of the given context and this subcontext.

2.6. Word Frequencies. We used two measures of D. melanogaster word frequencies. The first measure was obtained using complete aligned sequences of $37 \mathrm{D}$. melanogaster, the $D$. sechellia, and D. erecta genomes. For the second measure, we used conserved regions in which the ancestral nucleotide matches at least one of the D. melanogaster genetic variants, and no gaps or unread sequences are present in the multiple alignment. Word frequencies from the conserved regions were used for calculating mutation biases and contrasts. 
TABLE 1: Comparison of nucleotide composition of complete alignments and conserved regions of $D$. melanogaster.

\begin{tabular}{lccc}
\hline Nucleotide & $\begin{array}{c}\text { Nucleotide } \\
\text { fraction within } \\
\text { all positions }\end{array}$ & $\begin{array}{c}\text { Nucleotide } \\
\text { fraction within } \\
\text { conserved } \\
\text { positions }\end{array}$ & Difference, \% \\
\hline $\mathrm{a}$ & 0.2979 & 0.2901 & 2.6 \\
$\mathrm{t}$ & 0.2978 & 0.2899 & 2.7 \\
$\mathrm{c}$ & 0.2022 & 0.2100 & -3.9 \\
$\mathrm{~g}$ & 0.2021 & 0.2100 & -3.9 \\
\hline
\end{tabular}

\section{Results and Discussion}

The nucleotide composition of complete alignments and conserved regions (see Section 2) of D. melanogaster were similar (Table 1). We decided to use word frequencies within conserved regions of $D$. melanogaster for calculations of contrast and mutation bias.

Previous studies have shown that the representation of mutation data on a plot of mutation bias versus minimal contrast is useful for identifying important mutation contexts [10]. Mutation bias and minimal contrasts of mutation contexts in D. melanogaster are shown in Figure 1. The $\{\mathrm{A}>\mathrm{C}$ $\mid 2, \mathrm{CACC}\}$ and $\{\mathrm{A}>\mathrm{C} \mid 3, \mathrm{CCA}\}$ mutation contexts have the highest minimal contrast values in D. melanogaster. Interestingly, the addition of $\mathrm{C}$ or $\mathrm{G}$ nucleotides to either end of the word CCA increases mutation bias of the $\mathrm{A}>\mathrm{C}$ mutation, while the addition of $\mathrm{A}$ or $\mathrm{T}$ nucleotides to these words decreases mutation bias.

As shown in Table 2, mutation patterns differ between $D$. melanogaster and $H$. sapiens at the single nucleotide scale: D. melanogaster has a lower transition/transversion ratio. Moreover, the $\mathrm{G}>\mathrm{T}(\mathrm{C}>\mathrm{A})$ transversion in D. melanogaster comprises a much larger fraction of mutations than the $A>G$ $(\mathrm{T}>\mathrm{C})$ transition, which is consistent with previous findings [4].

One of the mechanisms by which $\mathrm{G}>\mathrm{T}(\mathrm{C}>\mathrm{A})$ transversions occur is through the formation of 8-Oxoguanine [12] caused by reactive oxygen species [13] or ultraviolet irradiation [14]. In eukaryotes, the damaged DNA is repaired with the help of DNA glycosylase OGG1. This enzyme removes the 8-oxoguanine, forming a DNA apurinic-apyrimidinic site, which is then recognized by other proteins of the DNA repair system. If further reparation does not occur, the apurinicapyrimidinic site will be complemented with an adenine nucleotide during DNA replication, resulting in a $\mathrm{C}>\mathrm{A}$ mutation. Another protein with DNA glycosylase activity for 8-hydroxyguanine, called dOgg1, was also described in $D$. melanogaster [15].

Another factor that might be responsible for increased $\mathrm{G}>\mathrm{T}(\mathrm{C}>\mathrm{A})$ transversion rates in $D$. melanogaster is aflatoxin $\mathrm{B} 1$. Aflatoxin $\mathrm{B} 1$ is known to induce base substitutions in DNA $[16,17]$, especially $\mathrm{G}>\mathrm{T}(\mathrm{C}>\mathrm{A})$ transversions. It is a product of a fungus from the Aspergillus genus, which grows on fruits and grains in a humid climate; thus, it is quite possible that $D$. melanogaster is exposed to this toxin.

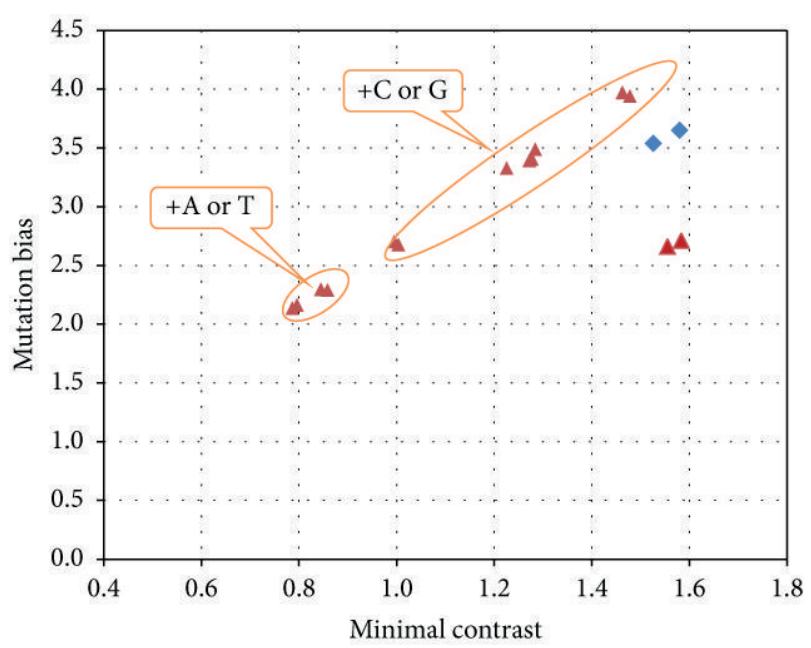

$$
\begin{aligned}
& \{A>C \mid 2, C A C C\} \\
\triangle & \{A>C \mid 3, C A C C\} \\
& \text { All }
\end{aligned}
$$

FIGURE 1: Mutation bias and minimal contrasts of mutation contexts in D. melanogaster. Each dot represents a mutation context. Triangles represent the $\{\mathrm{A}>\mathrm{C} \mid 3, \mathrm{CCA}\}$ (as well as complementary contexts) and contexts that had this context as a subcontext. Most dots are in pairs because complementary contexts have similar mutation bias and minimal contrast values.

TABle 2: Comparison of single nucleotide mutations in $D$. melanogaster and H. sapiens. Transitions are italic, while transversions are bold.

\begin{tabular}{lccc}
\hline \multicolumn{2}{c}{ D. melanogaster } & \multicolumn{2}{c}{ H. sapiens } \\
Mutation & Fraction & Mutation & Fraction \\
\hline A $>$ C & $\mathbf{0 , 0 4 4}$ & $\mathbf{A}>\mathbf{T}$ & $\mathbf{0 , 0 3 1}$ \\
T $>$ G & $\mathbf{0 , 0 4 7}$ & T $>\mathbf{A}$ & $\mathbf{0 , 0 3 1}$ \\
$\mathbf{C}>\mathbf{G}$ & $\mathbf{0 , 0 4 7}$ & $\mathbf{A}>\mathbf{C}$ & $\mathbf{0 , 0 3 7}$ \\
$\mathbf{G}>\mathbf{C}$ & $\mathbf{0 , 0 4 8}$ & T $>\mathbf{G}$ & $\mathbf{0 , 0 3 8}$ \\
$\mathbf{A}>\mathbf{T}$ & $\mathbf{0 , 0 5 7}$ & $\mathbf{C}>\mathbf{G}$ & $\mathbf{0 , 0 5 1}$ \\
T $>\mathbf{A}$ & $\mathbf{0 , 0 5 8}$ & $\mathbf{G}>\mathbf{C}$ & $\mathbf{0 , 0 5 1}$ \\
A $>G$ & 0,063 & $\mathbf{G}>\mathbf{T}$ & $\mathbf{0 , 0 5 8}$ \\
$T>C$ & 0,064 & $\mathbf{C}>\mathbf{A}$ & $\mathbf{0 , 0 5 8}$ \\
$\mathbf{G}>\mathbf{T}$ & $\mathbf{0 , 1 1 8}$ & $T>C$ & 0,118 \\
$\mathbf{C}>\mathbf{A}$ & $\mathbf{0 , 1 2 1}$ & $A>G$ & 0,118 \\
$C>T$ & 0,166 & $C>T$ & 0,204 \\
$G>A$ & 0,167 & $G>A$ & 0,204 \\
Transversions & $\mathbf{0 , 5 4 0}$ & Transversions & $\mathbf{0 , 3 5 5}$ \\
Transitions & 0,460 & Transitions & 0,645 \\
\hline
\end{tabular}

D. melanogaster and $H$. sapiens mutageneses are also strikingly different for several $2-4 \mathrm{bp}$ contexts, as shown in Figure 2. The $\{\mathrm{C}>\mathrm{T} \mid 1, \mathrm{CG}\},\{\mathrm{T}>\mathrm{C} \mid 2, \mathrm{ATTG}\}$, 


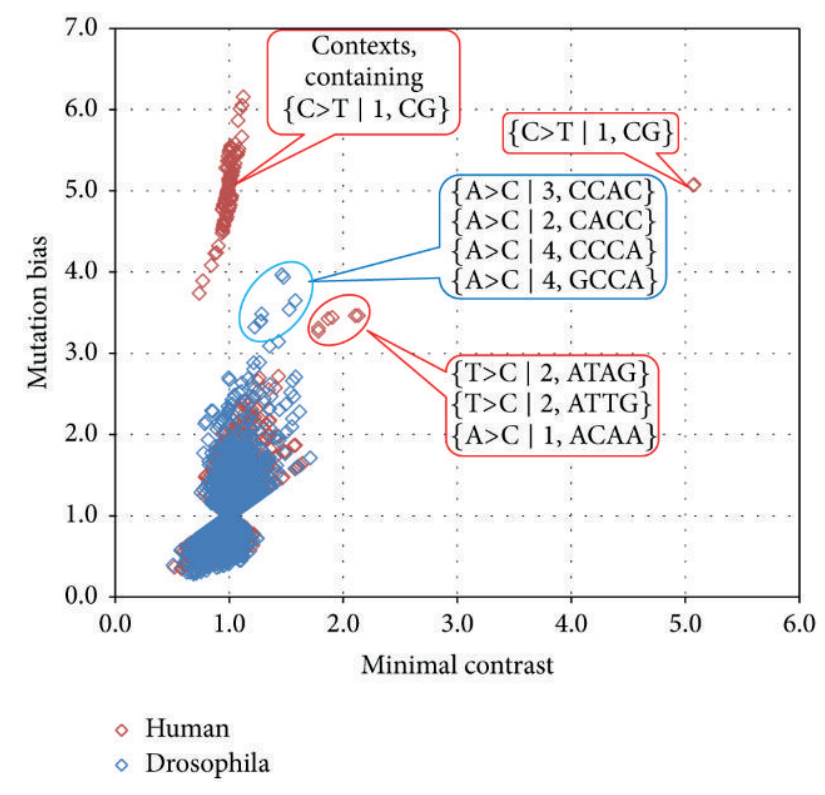

FIGURE 2: Mutation bias and minimal contrast for D. melanogaster and $H$. sapiens. Each dot represents a mutation context (blue in D. melanogaster, red in $H$. sapiens). Dots are overlapping and are usually in pairs because complementary contexts have similar mutation bias and minimal contrast values.

$\{\mathrm{T}>\mathrm{C} \mid 2, \mathrm{ATAG}\}$, and $\{\mathrm{A}>\mathrm{C} \mid 1, \mathrm{ACAA}\}$ mutation contexts appear to have excessive mutation frequencies in $\mathrm{H}$. sapiens but not in D. melanogaster. Interestingly, the CAATT sequence (contains the ATTG word on the reverse strand) appears to be a mutation hotspot for the human DNA polymerase eta [18]. Also, the CCAAT (contains the ATTG word on reverse strand) motif is a known target site for enhancer-binding proteins [19]. The increased number of ATTG > ACTG mutations might be partially due to selection against enhancers sequences in nontranscribed regions of the genome.

On the other hand, several mutation contexts seem to have increased mutation bias in $D$. melanogaster. The differences between different mutation contexts in $D$. melanogaster and $H$. sapiens are shown in more detail in Figure 3.

In a previous study, we compared the over- and underrepresentation of 1-7 bp nucleotide words in the genomes of 139 complete eukaryotic genomes, including $H$. sapiens and $D$. melanogaster [20]. Table 3 contains a part of this comparison for several words in $H$. sapiens and D. melanogaster related to the previously discussed mutation contexts. The word CG has a strong underrepresentation in $H$. sapiens (by $76.37 \%$ from the expected genomic frequency) while in D. melanogaster it is only slightly underrepresented (by $5.93 \%$ from the expected genomic frequency). The derived word TG is overrepresented by $20.1 \%$ and by $10.67 \%$ in $H$. sapiens and $D$. melanogaster, respectively. The $\{\mathrm{C}>\mathrm{T} \mid 1, \mathrm{CG}\}$ mutation context seems to be the only example of a mutation context that has remarkably affected the genomic word composition in $H$. sapiens compared to D. melanogaster. The absence of such effects for words related to other mutation contexts might be
TABLE 3: Over- and underrepresentation of genomic frequencies for several words in $H$. sapiens and D. melanogaster. Data is taken from a previous study [20] supplementary table (available at http://mouse.genebee.msu.ru/words/Supple3(contrast_k).xls). The numbers represent the value $C=[($ Obs $(W)-\operatorname{Exp}(W)) / \operatorname{Exp}$ $(\mathrm{W})] \cdot 100 \%$, where Obs (W) is the observed word frequency and Exp $(\mathrm{W})$ is the expected word frequency (based on the frequencies of all of its subwords).

Genomic word over- and underrepresentation in H. sapiens D. melanogaster

Words containing a mutation context with increased mutation bias in H. Sapiens

$\begin{array}{lcc}\text { CG } & -76.37 \% & -5.93 \% \\ \text { ATAG } & -0.79 \% & 4.38 \% \\ \text { ATTG } & -7.07 \% & -2.35 \% \\ \text { ACAA } & 1.62 \% & 3.75 \%\end{array}$

Words derived from mutation contexts with increased mutation bias in H. Sapiens

$\begin{array}{lcc}\text { TG } & 20.10 \% & 10.67 \% \\ \text { ACAG } & 1.51 \% & -4.94 \% \\ \text { ACTG } & -2.07 \% & -0.46 \% \\ \text { CCAA } & -6.17 \% & -1.61 \%\end{array}$

Words containing mutation contexts with increased mutation bias in D. melanogaster

$\begin{array}{lcc}\text { CCAC } & 0.19 \% & 1.52 \% \\ \text { CACC } & 1.18 \% & -4.24 \% \\ \text { CCCA } & 5.63 \% & 0.09 \% \\ \text { GCCA } & -2.77 \% & 3.63 \% \\ \text { ACC } & 2.28 \% & -2.39 \% \\ \text { CCA } & 14.82 \% & 9.90 \%\end{array}$

Words derived from mutation contexts with increased mutation bias in D. melanogaster

\begin{tabular}{lcc} 
CCCC & $-5.10 \%$ & $2.19 \%$ \\
GCCC & $1.66 \%$ & $-1.41 \%$ \\
CCC & $-12.66 \%$ & $-7.78 \%$ \\
\hline
\end{tabular}

due to us not taking into account the rates of other mutations in these words or mutations that produce these words.

\section{Conclusions}

The regularities of mutagenesis are different in D. melanogaster and H. sapiens. However, these differences may be attributed to a rather small number of mutation contexts that behave in a different manner in these two species. First, there is an increased frequency of $\mathrm{G}>\mathrm{T}(\mathrm{C}>\mathrm{A})$ transversions in $D$. melanogaster. Several possible molecular mechanisms for this have been proposed. Second, there is an increased frequency 


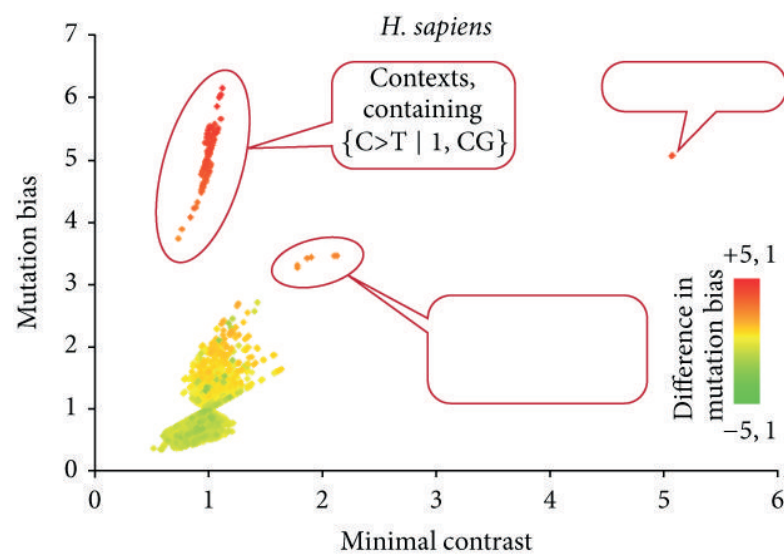

(a)

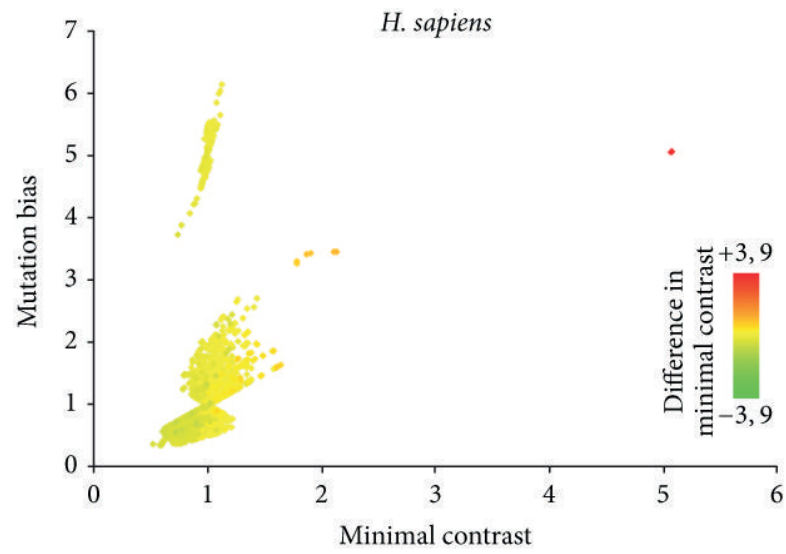

(c)

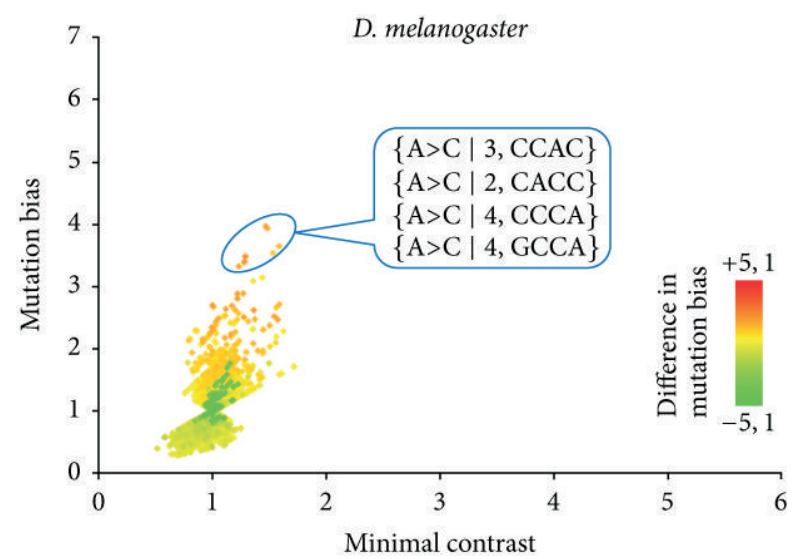

(b)

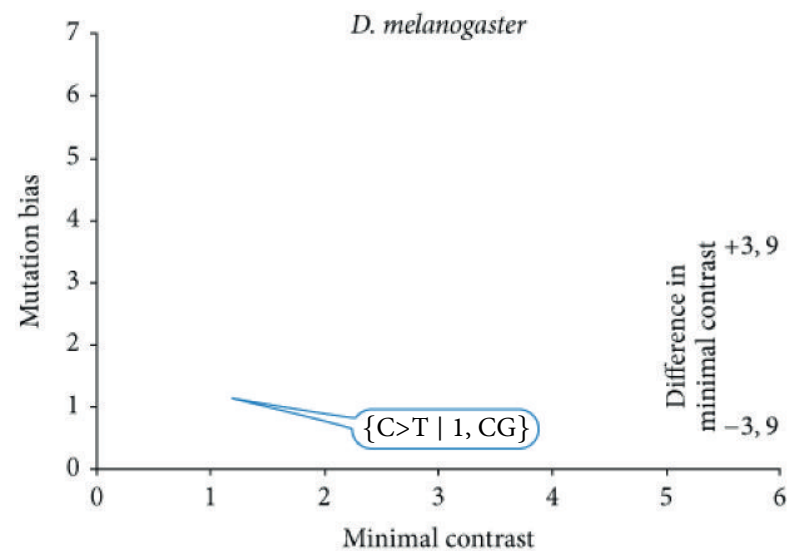

(d)

Figure 3: The difference between H. sapiens and D. melanogaster mutation bias ((a) and (b)) and minimal contrast ((c) and (d)) for 2$4 \mathrm{bp}$ mutation contexts. Each dot represents a mutation context. The $X$ axis represents the contexts minimal contrast values, and the $Y$ axis represents the contexts mutation bias. The minimal contrast and mutation bias values are given for H. sapiens ((a) and (c)) and for D. melanogaster ((b) and (d)), and the color scheme indicates the difference between minimal contrasts. Thus, red dots on (a) and (c) represent contexts that are hypermutable in humans comparing to drosophila, while green dots represent contexts that are hypermutable in D. melanogaster comparing to H. sapiens. This scheme is reversed for (b) and (d).

of $\mathrm{C}>\mathrm{T}$ mutation in the word CG in H. sapiens. This is probably explained by the fact that human germline methylation is abundant and CpG specific, while D. melanogaster is not. Third, there is an increased frequency of $\mathrm{T}>\mathrm{C}$ mutations in the second position of the words ATTG and ATAG and an increased frequency of $\mathrm{A}>\mathrm{C}$ mutations in the first position of the ACAA word in $H$. sapiens but not in D. melanogaster. And finally, there is an increased $A>C$ mutations rate in $\{A>C$ $\mid 2, C A C C\}$ and $\{A>C \mid 3, C C A\}$ mutation contexts in $D$. melanogaster but not in $H$. sapiens.

\section{Acknowledgments}

This work was supported by Russian Ministry of Science and Education State Contracts 8494 and 8100 of the Federal Special Program "Scientific and Educational Human Resources of Innovative Russia" for 2009-2013 and the Russian Foundation for Basic Research Grants 12-04-91334, 11-04-91340, 1307-00969, 12-04-31071, and 11-04-01511.

\section{References}

[1] C. F. Baer, M. M. Miyamoto, and D. R. Denver, "Mutation rate variation in multicellular eukaryotes: causes and consequences," Nature Reviews Genetics, vol. 8, no. 8, pp. 619-631, 2007.

[2] A. Kong, M. L. Frigge, G. Masson et al., "Rate of de novo mutations and the importance of father's age to disease risk," Nature, vol. 488, no. 7412, pp. 471-475, 2012.

[3] D. N. Cooper and M. Krawczak, "Cytosine methylation and the fate of CpG dinucleotides in vertebrates genomes," Human Genetics, vol. 83, no. 2, pp. 181-188, 1989.

[4] N. D. Singh, P. F. Arndt, A. G. Clark, and C. F. Aquadro, "Strong evidence for lineage and sequence specificity of substitution rates and patterns in drosophila," Molecular Biology and Evolution, vol. 26, no. 7, pp. 1591-1605, 2009.

[5] F. Lyko, B. H. Ramsahoye, and R. Jaenisch, "DNA methylation in Drosophila melanogaster," Nature, vol. 408, no. 6812, pp. 538540, 2000.

[6] N. Arnheim and P. Calabrese, "Understanding what determines the frequency and pattern of human germline mutations," Nature Reviews Genetics, vol. 10, no. 7, pp. 478-488, 2009. 
[7] A. Hodgkinson, E. Ladoukakis, and A. Eyre-Walker, "Cryptic variation in the human mutation rate," PLoS Biology, vol. 7, no. 2, Article ID e1000027, 2009.

[8] R. D. Blake, S. T. Hess, and J. Nicholson-Tuell, "The influence of nearest neighbors on the rate and pattern of spontaneous point mutations," Journal of Molecular Evolution, vol. 34, no. 3, pp. 189-200, 1992.

[9] D. G. Hwang and P. Green, "Bayesian Markov chain Monte Carlo sequence analysis reveals varying neutral substitution patterns in mammalian evolution," Proceedings of the National Academy of Sciences of the United States of America, vol. 101, no. 39, pp. 13994-14001, 2004.

[10] A. Y. Panchin, S. I. Mitrofanov, A. V. Alexeevski, S. A. Spirin, and Y. V. Panchin, "New words in human mutagenesis," BMC Bioinformatics, vol. 12, article 268, 2011.

[11] R. M. Kuhn, D. Karolchik, A. S. Zweig et al., "The UCSC genome browser database: update 2009," Nucleic Acids Research, vol. 37, no. 1, pp. D755-D761, 2009.

[12] K. C. Cheng, D. S. Cahill, H. Kasai, S. Nishimura, and L. A. Loeb, "8-Hydroxyguanine, an abundant form of oxidative DNA damage, causes $\mathrm{G} \rightarrow \mathrm{T}$ and $\mathrm{A} \rightarrow \mathrm{C}$ substitutions," Journal of Biological Chemistry, vol. 267, no. 1, pp. 166-172, 1992.

[13] D. Wang, D. A. Kreutzer, and J. M. Essigmann, "Mutagenicity and repair of oxidative DNA damage: insights from studies using defined lesions," Mutation Research, vol. 400, no. 1-2, pp. 99-115, 1998.

[14] T. Douki, D. Perdiz, P. Gróf et al., "Oxidation of guanine in cellular DNA by solar UV radiation: biological role," Photochemistry and Photobiology, vol. 70, no. 2, pp. 184-190, 1999.

[15] C. Dherin, M. Dizdaroglu, H. Doerflinger, S. Boiteux, and J. P. Radicella, "Repair of oxidative DNA damage in Drosophila melanogaster: identification and characterization of dOggl, a second DNA glycosylase activity for 8-hydroxyguanine and formamidopyrimidines," Nucleic Acids Research, vol. 28, no. 23, pp. 4583-4592, 2000.

[16] P. L. Foster, E. Eisenstadt, and J. H. Miller, "Base substitution mutations induced by metabolically activated aflatoxin B1," Proceedings of the National Academy of Sciences of the United States of America, vol. 80, no. 9, pp. 2695-2698, 1983.

[17] Y. Trottier, W. I. Waithe, and A. Anderson, "Kinds of mutations induced by aflatoxin B1 in a shuttle vector replicating in human cells transiently expressing cytochrome P450IA2 cDNA," Molecular Carcinogenesis, vol. 6, no. 2, pp. 140-147, 1992.

[18] T. Matsuda, K. Bebenek, C. Masutani, I. B. Rogozin, F. Hanaoka, and T. A. Kunkel, "Error rate and specificity of human and murine DNA polymerase $\eta$," Journal of Molecular Biology, vol. 312, no. 2, pp. 335-346, 2001.

[19] S. Osada, H. Yamamoto, T. Nishihara, and M. Imagawa, "DNA binding specificity of the CCAAT/enhancer-binding protein transcription factor family," Journal of Biological Chemistry, vol. 271, no. 7, pp. 3891-3896, 1996.

[20] S. I. Mitrofanov, A. Y. Panchin, S. A. Spirin, A. V. Alexeevski, and Y. V. Panchin, "Exclusive sequences of different genomes," Journal of Bioinformatics and Computational Biology, vol. 8, no. 3, pp. 519-534, 2010. 

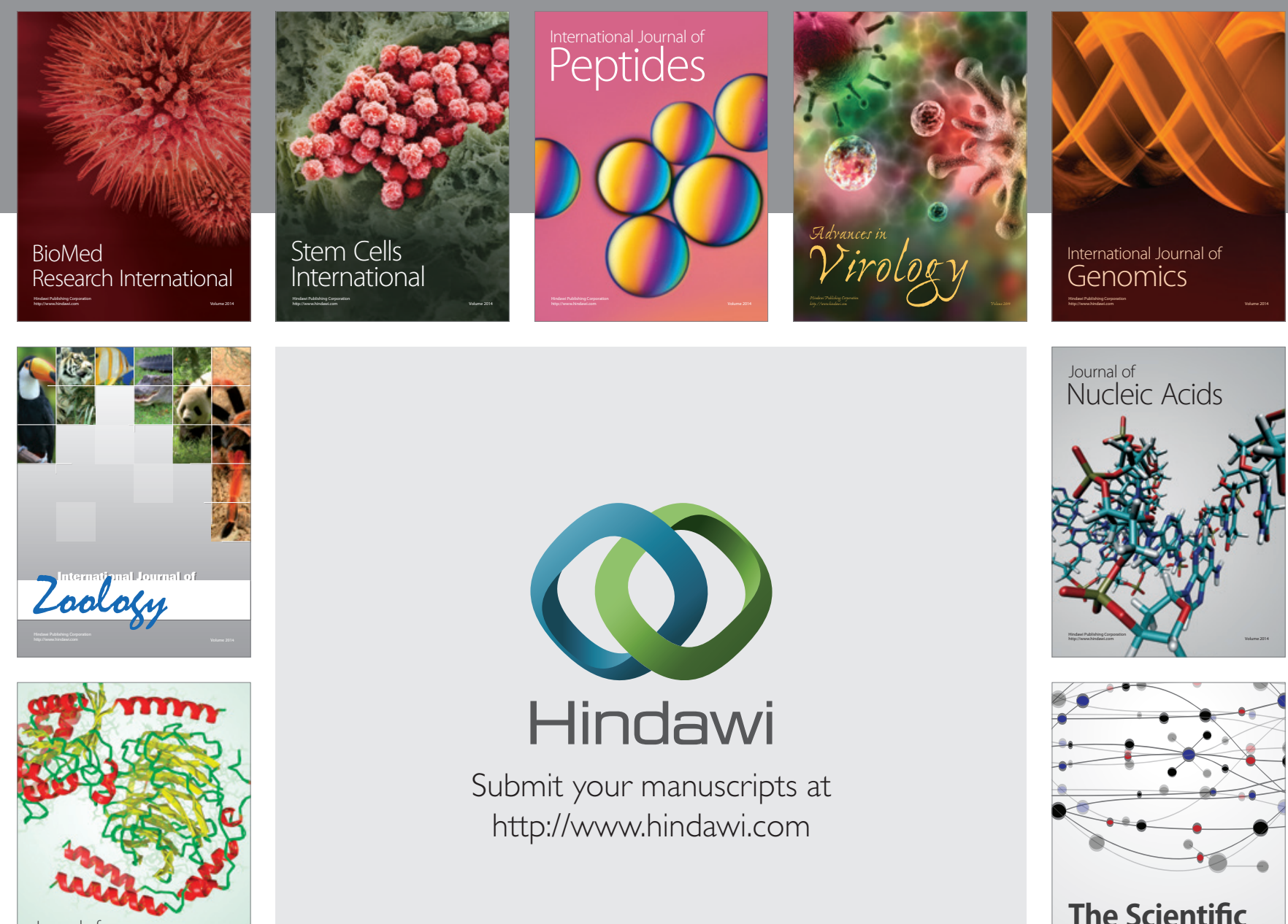

Submit your manuscripts at

http://www.hindawi.com

Journal of
Signal Transduction
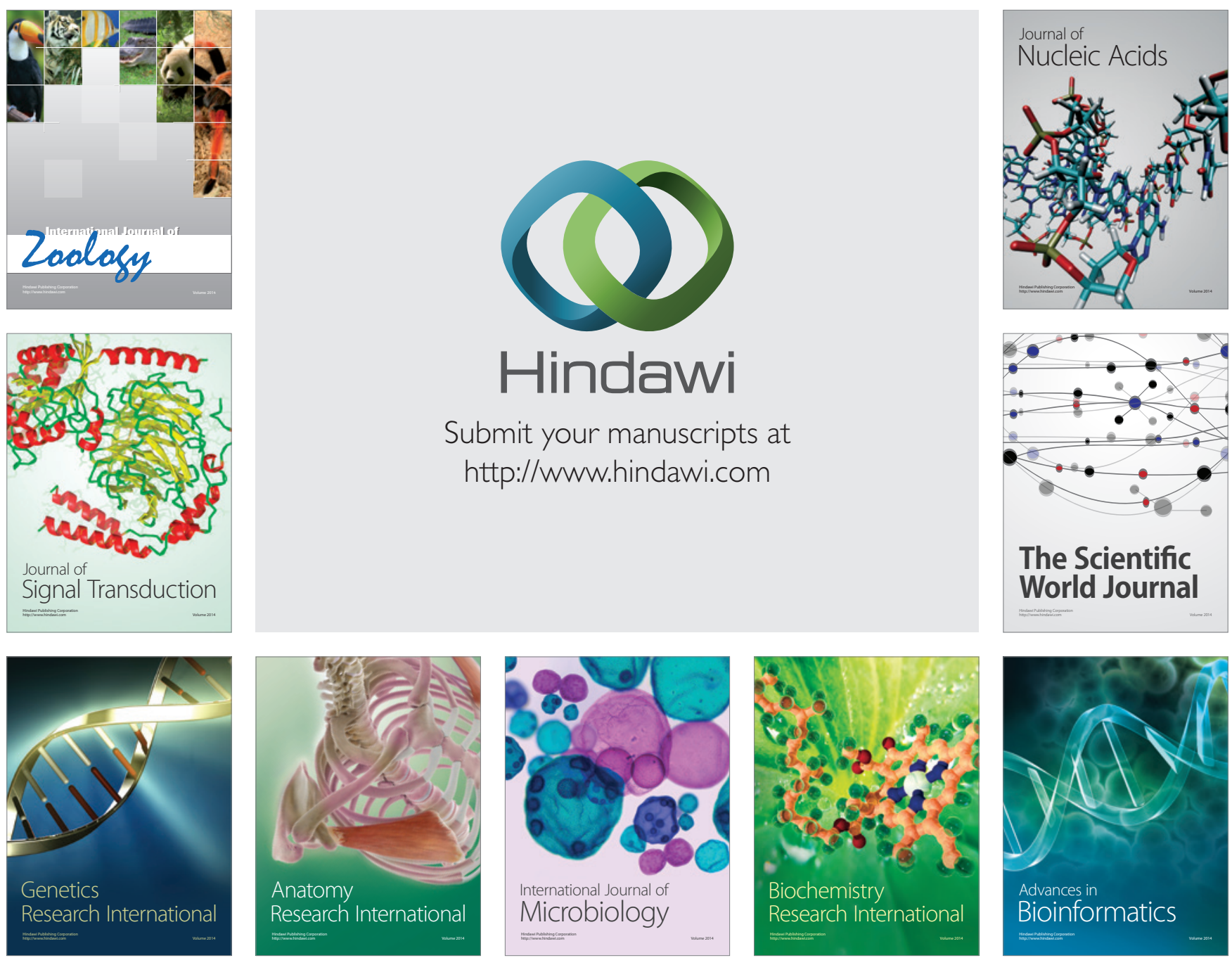

The Scientific World Journal
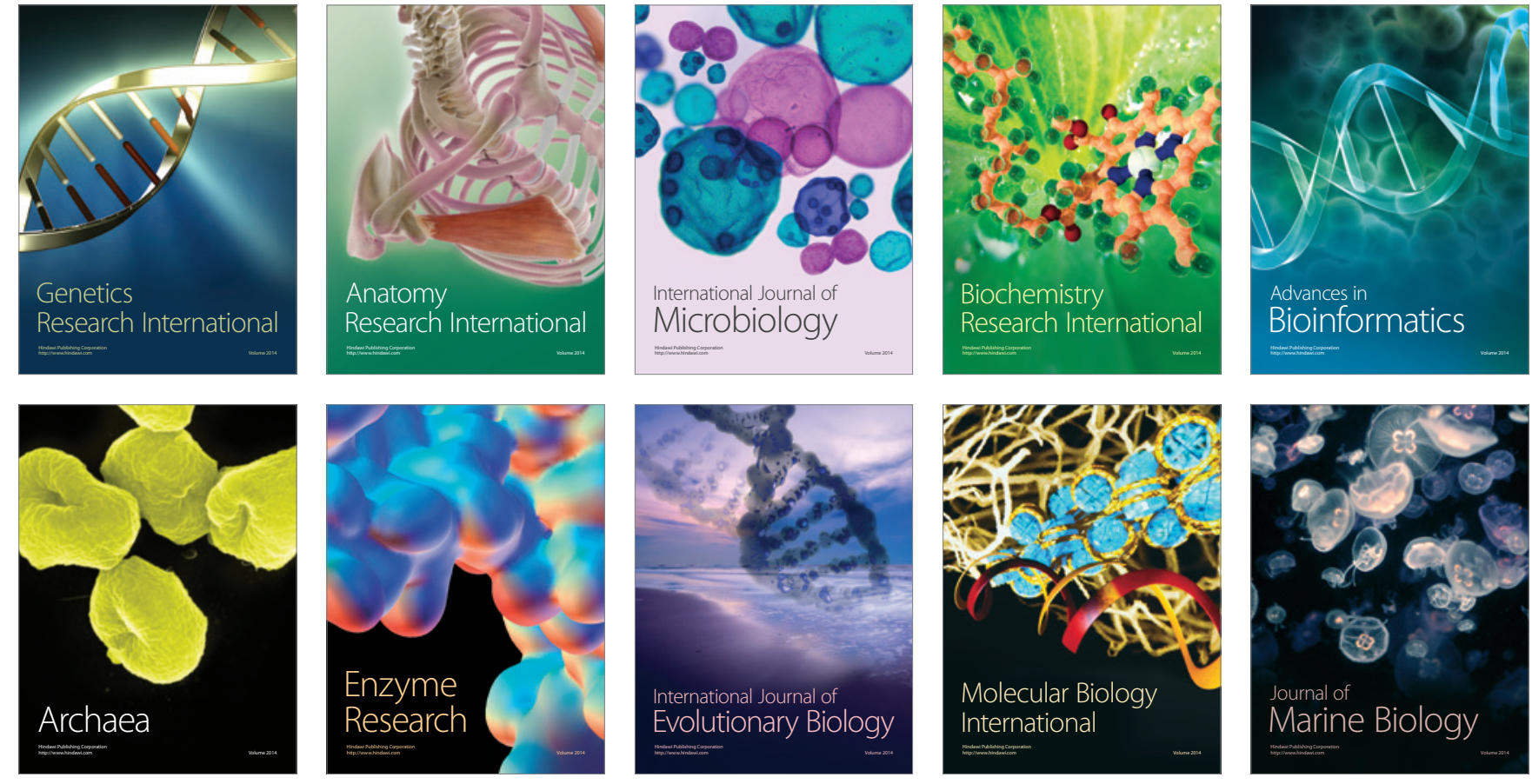